\title{
Ventricular Standstill Following Intravenous Erythromycin and Borderline Hypokalemia
}

\author{
$\underline{\text { Saad Khan }}{ }^{1}$, John Ramzy², Danae Papachristos ${ }^{3}$, Nayana George ${ }^{1}$, Leon Fisher ${ }^{1}$ \\ ${ }^{1}$ Department of Gastroenterology, Peninsula Health, Frankston, Australia \\ ${ }^{2}$ Department of Medicine, Eastern Health, Box Hill, Australia \\ ${ }^{3}$ Department of Medicine, St Vincent's Health, Fitzroy, Australia
}

Received: 04/01/2015

Accepted: 14/01/2015

Published: $17 / 02 / 2016$

How to cite this article: Khan S, Ramzy J, Papachristos D, George N, Fisher L. Ventricular standstill following intravenous erythromycin and borderline hypokalemia. EJCRIM 2016;3:doi:10.12890/2016_000375

Conflicts of Interests: The authors declare that there are no competing interests.

This article is licensed under a Commons Attribution Non-Commercial 4.0 License

\section{ABSTRACT}

Ventricular standstill (VS) is a potentially fatal arrhythmia that is usually associated with syncope, if prolonged and is rarely asymptomatic ${ }^{[1]}$. Its mechanism involves either a lack of supraventricular impulse or an interruption in the transmission of these signals from the atria to the ventricles, resulting in a sudden loss of cardiac output ${ }^{[2]}$. Although rare, ventricular arrhythmias have been associated with intravenous (IV) erythromycin. However, to our knowledge, VS has not been reported following the administration of IV erythromycin. The Authors describe a rare case of asymptomatic VS and subsequent third-degree atrioventricular block, following the administration of IV erythromycin in a 49-year-old woman with borderline hypokalemia. Through this case, the Authors highlight the importance of cardiac monitoring and electrolyte replacement when administering IV erythromycin, as well as discuss several other mechanisms that contribute to ventricular arrhythmias.

\section{LEARNING POINTS}

- Intravenous erythromycin is associated with prolongation of the QTc interval and ventricular arrhythmias.

- Ventricular standstill is a rare but potentially fatal arrhythmia, and may have an association with the administration of intravenous erythromycin.

- Cardiac monitoring in patients with baseline QTc prolongation and correction of electrolyte disturbances are important when administering intravenous erythromycin.

\section{KEYWORDS}

Arrhythmias; ventricular standstill; erythromycin.

\section{CASE REPORT}

A 49-year-old woman with a past medical history of type 2 diabetes, hypertension and migraines presented to a regional hospital with symptoms suggestive of viral gastroenteritis and secondary atypical pneumonia. She did not have any known drug allergies. The patient described a five-day history of generalized abdominal pain, associated diarrhea and vomiting, as well as a two-day history of productive cough, mild dyspnea, and coryzal symptoms. 
Her vital signs on admission were as follows: temperature $36.8^{\circ} \mathrm{C}$; heart rate $80 / \mathrm{min}$ regular; oxygen saturation $97 \%$ on room air; and blood pressure 120/75 mmHg. Her body mass index(BMI) was $26.2 \mathrm{~kg} / \mathrm{m}^{2}$. Abdominal examination revealed mild generalised tenderness, but was soft with normal bowel sounds. Cardiovascular and respiratory examinations were unremarkable. Baseline 12-lead electrocardiogram (ECG) showed normal sinus rhythm. There was evidence of bilateral peri-hilar and infra-hilar opacities on chest x-ray, suggestive of atypical pneumonia. Initial biochemistry was unremarkable except for an elevated c-reactive protein of $89.6 \mathrm{mg} / \mathrm{L}$ (reference range [RR], < $10 \mathrm{mg} / \mathrm{L}$ ). In particular, her electrolytes were within normal parameters, though a borderline hypokalemia was noted (magnesium 0.72mmol/L [RR, 0.6-1.1 mmol/L], potassium 3.5mmol/L [RR, 3.5-5 mmol/L). Fecal culture and clostridium difficile toxin were negative.

She was initially commenced on regulation doses of oral amoxicillin and doxycycline for a mild community-acquired pneumonia. These were ceased on day 1 of her admission. She was subsequently commenced on IV erythromycin 500mg 6-hourly and was monitored with telemetry for significant QT prolongation. She received three doses in total, at the times 10:00, 16:00 and 22:00. During sleep, at 00:34 on day 2 of admission (approximately 2.5 hours after her third dose of IV erythromycin), a seven second period of VS was noted on cardiac monitoring (Fig. 1). This rhythm then progressed to a third-degree atrioventricular (AV) block (Fig. 2). This persisted for approximately 2 minutes then returned to normal sinus rhythm. The patient remained hemodynamically stable and asleep throughout this entire period.

She was in normal sinus rhythm leading up to VS, with no variations in the PR-interval. The rate-corrected QT interval (QTc) prior to erythromycin administration was 410 milliseconds (ms). The QTc following erythromycin administration and immediately prior to the period of VS was 420 ms.

Erythromycin was discontinued following this event and a specialist cardiology opinion was sought. Magnesium and potassium were replaced intravenously. A further 36-hour period of cardiac monitoring did not reveal any further episodes of VS and thus no further action was taken. There was significant improvement in the patient's gastrointestinal and respiratory symptoms and she was subsequently discharged home with oral doxycycline. Outpatient holter monitor did not reveal any cardiac arrhythmias. She remained well at six-month follow-up.

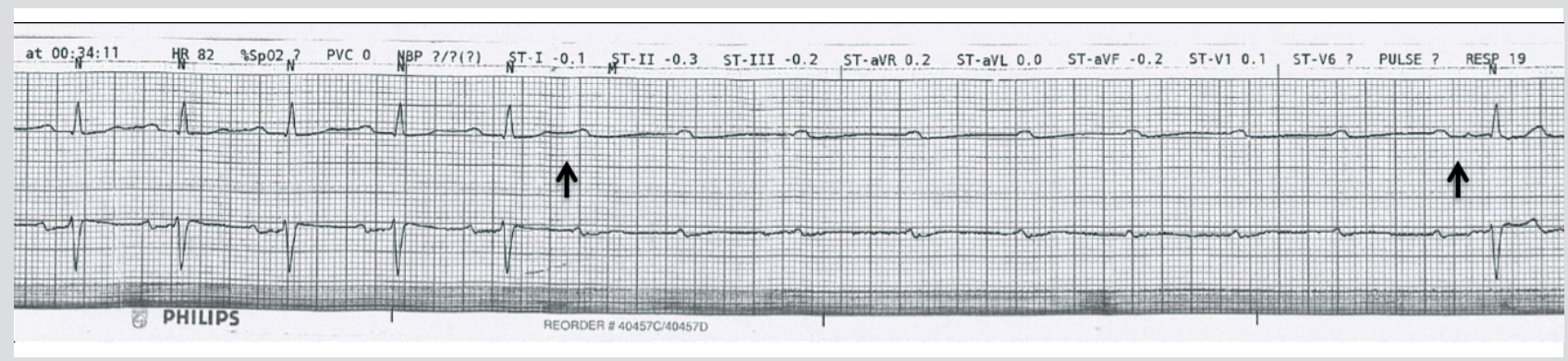

Figure 1: Printout from cardiac monitor showing normal sinus rhythm, and then sudden onset of ventricular standstill lasting approximately 7 seconds (between arrows), characterized by regular $p$-waves but the absence of subsequent QRS complexes.

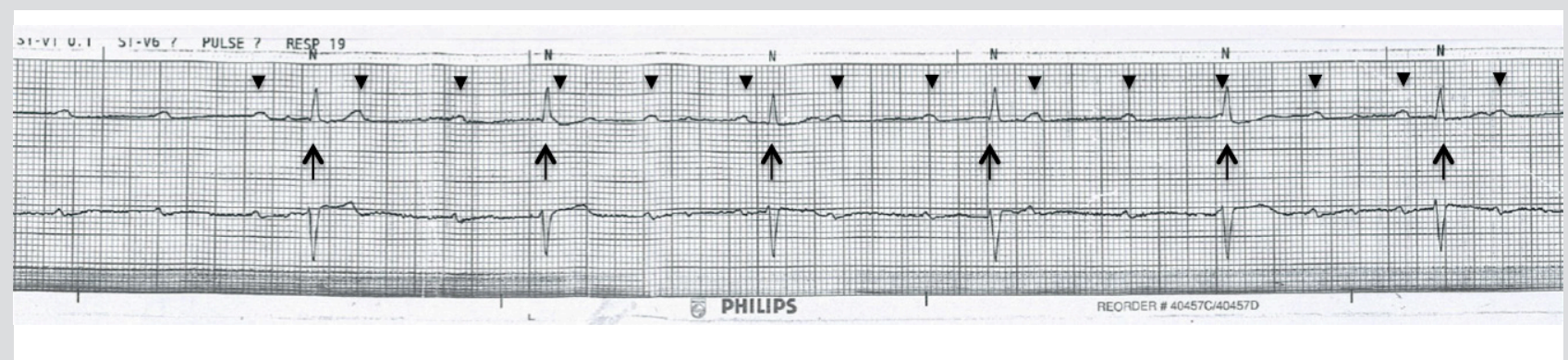

Figure 2: Printout from cardiac monitor showing progression of VS into third-degree AV block. This is characterized by the presence of $p$-waves with regular $p$-to $p$-intervals (arrowheads), and independent QRS complexes with regular R- to R-intervals (arrows), but no apparent relationship between $p$-waves and QRS complexes. 
Her vital signs on admission were as follows: temperature $36.8^{\circ} \mathrm{C}$; heart rate $80 /$ min regular; oxygen saturation $97 \%$ on room air; and blood pressure 120/75 mmHg. Her body mass index (BMI) was $26.2 \mathrm{~kg} / \mathrm{m} 2$. Abdominal examination revealed mild generalised tenderness, but was soft with normal bowel sounds. Cardiovascular and respiratory examinations were unremarkable. Baseline 12-lead electrocardiogram (ECG) showed normal sinus rhythm. There was evidence of bilateral peri-hilar and infra-hilar opacities on chest x-ray, suggestive of atypical pneumonia. Initial biochemistry was unremarkable except for an elevated c-reactive protein of $89.6 \mathrm{mg} / \mathrm{L}$ (reference range [RR], < 10 mg/L). In particular, her electrolytes were within normal parameters, though a borderline hypokalemia was noted (magnesium 0.72mmol/L [RR, 0.6-1.1 mmol/L], potassium 3.5mmol/L [RR, 3.5-5 mmol/L). Fecal culture and clostridium difficile toxin were negative.

She was initially commenced on regulation doses of oral amoxicillin and doxycycline for a mild community-acquired pneumonia. These were ceased on day 1 of her admission. She was subsequently commenced on IV erythromycin 500mg 6-hourly and was monitored with telemetry for significant QT prolongation. She received three doses in total, at the times 10:00, 16:00 and 22:00. During sleep, at 00:34 on day 2 of admission (approximately 2.5 hours after her third dose of IV erythromycin), a seven second period of VS was noted on cardiac monitoring (Fig. 1). This rhythm then progressed to a third-degree atrioventricular (AV) block (Fig. 2). This persisted for approximately 2 minutes then returned to normal sinus rhythm. The patient remained hemodynamically stable and asleep throughout this entire period.

She was in normal sinus rhythm leading up to VS, with no variations in the PR-interval. The rate-corrected QT interval (QTC) prior to erythromycin administration was 410 milliseconds ( $\mathrm{ms}$ ). The QTc following erythromycin administration and immediately prior to the period of VS was 420 ms.

Erythromycin was discontinued following this event and a specialist cardiology opinion was sought. Magnesium and potassium were replaced intravenously. A further 36-hour period of cardiac monitoring did not reveal any further episodes of VS and thus no further action was taken. There was significant improvement in the patient's gastrointestinal and respiratory symptoms and she was subsequently discharged home with oral doxycycline. Outpatient holter monitor did not reveal any cardiac arrhythmias. She remained well at six-month follow-up.

\section{DISCUSSION}

The mechanism of VS involves either a lack of supraventricular impulse or an interruption in the transmission of these signals from the atria to the ventricles, resulting in a sudden loss of cardiac output ${ }^{[2]}$. The usual ECG appearance of VS is the absence of QRS complexes despite the presence of regular p-waves, with or without occasional ventricular escape rhythms ${ }^{[1]}$. This was observed in our patient during cardiac monitoring. VS is commonly associated with dizziness, syncope and cardiac arrest, if prolonged; however asymptomatic VS, as in our case, is extremely rare.

Rapid eye movement (REM) sleep has been associated with transient bradyarrhythmias and AV blockade. It has, thus, been postulated that episodes of VS may be attributable to the vagotonic effects of REM sleep ${ }^{[3]}$. This may have been a contributing factor in our case; however, this mechanism is not well understood or documented. Transient AV blockade secondary to increased vagal tone has also been described in many situations, including vomiting ${ }^{[4]}$. Our patient had not vomited for over 12 hours prior to the time of her VS with prolonged AV block and thus, it is unlikely to have been a factor. Obstructive sleep apnea (OSA) has also been associated with cardiac arrhythmias and conduction disturbances. While our patient had a BMI slightly in the overweight range, she did not describe any symptoms suggestive of OSA.

Although rare, ventricular arrhythmias associated with IV erythromycin have been well documented. However, these usually occur in the setting of abnormal cardiac electrophysiology, such as electrolyte disturbances or bradycardia ${ }^{[5]}$. Our patient was found to be borderline hypokalemic prior to the event, and though it is unclear if she was hypokalemic at the time of VS, this may have been a contributing factor. An important mechanism of transient hypokalemia in an acutely ill patient is the intracellular shift of potassium caused by endogenous catecholamines acting on beta-2 adrenergic receptors ${ }^{[6]}$. In conjunction with vomiting and diarrhea, this mechanism may have contributed to our patient's hypokalemia.

IV erythromycin is well known to be associated with prolongation of the rate-corrected QT interval (QTC) on ECG, as well as its potential consequence, polymorphic ventricular tachycardia (torsades de pointes). This effect appears to be further associated with infusion rate ${ }^{[7]}$ and its arrhythmogenic potential appears to be greater than other QTc prolonging antibiotics, such as fluoroquinolones ${ }^{[8]}$. The Authors estimate from the rhythm strip of the cardiac monitoring that the patient's QTc prolonged by approximately 10 ms as a result of erythromycin; however, the QTc remained within normal limits at all times. 
We feel that given the onset of VS and subsequent prolonged third-degree AV blockade following administration, as well as the lack of recurrence after cessation, IV erythromycin may have played a role in the mechanism of this patient's ventricular arrhythmia. Cardiac arrhythmias secondary to antibiotics in the absence of QTc prolongation is extremely rare, and to our knowledge, VS following IV erythromycin has not previously been reported. Patients with baseline QTc prolongation should, however, be stratified as higher risk and prioritized for cardiac monitoring. Further investigation is required to better outline any potential association between IV erythromycin and VS. Our case also highlights the importance of electrolyte correction when administering medications that are associated with cardiac arrhythmias.

\section{REFERENCES}

1. Vassalle M. On the mechanisms underlying cardiac standstill: factors determining success or failure of escape pacemakers in the heart. J Am Coll Cardiol 1985;5:35B-42B.

2. Lynch RM, Ballesty L, Maicoo R. Be still my beating heart: Ventricular standstill occurring in difference age groups. Afr J Emerg Med 2014;4:e12-e15.

3. Serafini A, Dolso P, Gigli GL, Fratticci, Cancelli I, Facchin D, et al. REM sleep brady-arrhythmias: An indication to pacemaker implantation? Sleep Med 2012;13:759-62.

4. Jaiswal S, Aldave APN, Wool KJ. Ventricular standstill: An uncommon electrophysiological abnormality caused by profound vagal tone. N Am J Med Sci $2014 ; 6: 178$.

5. Oberg KC, Bauman JL. QT interval prolongation and torsades de pointes due to erythromycin lactobionate. Pharmacotherapy 1995;15:687-692.

6. Brown MJ, Brown DC, Murphy MB. Hypokalemia from beta2-receptor stimulation by circulating epinephrine. N Engl J Med 1983;309:1414-1419

7. Haefeli WE, Schoenenberger RA, Weiss PH, Ritz R. Possible risk of cardiac arrhythmia related to intravenous erythromycin. Intensive Care Med 1992;18:469-473.

8. Wisialowski T, Crimin K, Engtrakul J, O'Donnell J, Fermini B, Fossa AA. Differentiation of arrhythmia risk of the antibacterials moxifloxacin, erythromycin, and telithromycin based on analysis of monophasic action potential duration alternans and cardiac instability. J Pharmacol Exp Ther 2006;318:352-359. 